\title{
Effect of Thermomechanical History on the Crystallization of Poly(ether-block-amide)
}

\author{
Bruno Tavernier, ${ }^{1}$ Jan Mewis, ${ }^{1}$ Peter Van Puyvelde, ${ }^{1}$ Mikihito Takenaka, ${ }^{2}$ Benoît Ernst, ${ }^{3}$ \\ Takeji Hashimoto ${ }^{4}$ \\ ${ }^{1}$ K.U. Leuven, Department of Chemical Engineering, W. de Croylaan 46, Leuven B-3001, Belgium \\ ${ }^{2}$ Department of Polymer Chemistry, Graduate School of Engineering, Kyoto University, Nishikyo-Ku, \\ Kyoto 615-8510, Japan
}

${ }^{3}$ ARKEMA, Centre d'Etude de Recherche et Développement, 27470 Serquigny, France

${ }^{4}$ Advanced Science Research Center, Japan Atomic Energy Agency, Tokaimura, Ibaraki Pref 319-1195, Japan

\begin{abstract}
The quiescent and flow-induced crystallization of a poly(ether-block-amide) is studied by means of rheooptical methods. Both optical microscopy and small angle light scattering have been used. The multiblock copolymer has a microphase-separated structure with an order-disorder transition at $180-185^{\circ} \mathrm{C}$, as measured with rheometry and SAXS. The number of nuclei, spherulitic growth rates, and the characteristic time scale for crystallization are compared with that of a polyamide of similar molar mass. For the poly(etherblock-amide)-containing a majority of amide segments-the growth rates of the spherulites during quiescent crystallization are similar for the block copolymer and the homopolymer, even if the spherulitic structures are not the same. When flow is applied, the two materials behave differently. The flow increased the nucleation density in the homopolymer but not in the block copolymer. POLYM. ENG. SCI., 48:2418-2425, 2008. () 2008 Society of Plastics Engineers
\end{abstract}

\section{INTRODUCTION}

Thermoplastic elastomers constitute an interesting class of materials. Such materials are typically composed of alternating, flexible soft and more rigid segments. By tuning the relative length of these segments during synthesis, materials can be prepared with properties ranging from soft elastomers to hard rubbers [1], a versatility that has inspired many industrial applications. The hard, often crystalline, segments act as virtual crosslinks between the more flexible soft seg-

Correspondence to: Peter Van Puyvelde; e-mail: peter.vanpuyvelde@cit. kuleuven.be

Contract grant sponsor: Arkema.

DOI 10.1002/pen.21197

Published online in Wiley InterScience (www.interscience.wiley.com) (C) 2008 Society of Plastics Engineers ments. Hence, unlike conventional rubbers that contain covalent crosslinks, the physical crosslinks used in thermoplastic elastomers can be melted, allowing the polymer to be processed as a conventional thermoplastic.

Immiscibility of the hard and soft blocks can give rise to microphase separations on a very local scale. Such phenomena have already been observed in materials as thermoplastic polyurethanes [2-4]. The dimensions of the structural elements that result from this segmental repulsion are usually of the order of the dimensions of the macromolecule [1]. The type and size of the thus-obtained microdomains are a function of the relative block lengths. They can vary from spheres of the minority component, when the composition is very asymmetric, to alternating lamellar morphologies for symmetric copolymers [5-9].

In this work, a multiblock copolymer of the type poly (ether-block-amide) will be investigated. This copolymer has good mechanical properties and can be used to produce molded or extruded products such as films, sheets, and fibers. The copolymers are currently used in a variety of applications such as sport equipment, toys, automotive, medical supplies, and others. Despite a wide range of applications, very little is known about the thermodynamic properties of these copolymers $[10,11]$. Di Lorenzo et al. [11] studied the crystallization, vitrification, and melting behavior of various samples and their dependence on composition and molar mass of the blocks. In all compositions investigated, the copolymer presented two separate glass transitions, rather close to those of the corresponding homopolymers. The amide segments crystallized from the melt with a significant degree of crystallinity, whereas the ether segments solidified later in the presence of crystals and glass of the second component. 
Because of the highly reduced mobility of the ether chain segments, their crystallinity was rather small.

The morphology development of this material has been studied by transmission electron [12] and atomic force microscopy [13-15], small and wide angle X-ray scattering $[15,16]$, small angle light scattering (SALS) [15], DSC [11, 12], as well as by dynamical mechanical analysis [17]. The investigations showed consistent results, indicating that the polymer exhibited a multiphase morphology that included a well-defined polyamide crystalline phase, a polyamide amorphous phase, and a polyether amorphous phase.

Most of the available studies focused on the morphology analysis in the solid state in order to elucidate structure-property relations of the final product, without considering the preceding processing and solidification steps. The structure and, therefore, also its final properties depend to a large extent on the thermomechanical history the material is subjected to during processing and solidification. Understanding the impact of these steps on the structure is of crucial importance in order to tailor the final properties of the material. To process the material properly, the effect of the rheological behavior on the processing (i.e., flow) and solidification steps has to be understood. At present, the rheological behavior of poly (ether-block-amide) has only been investigated in a few cases [18, 19], mostly focusing on the detection of the order-disorder temperature. No studies seem to be available that deal with the effect of flow on the crystallization behavior of poly(ether-block-amide) or of segmented multiblock copolymers in general.

In homopolymers, flow can drastically influence the crystallization process [20, 21]. For instance, it is known that the nucleation density and nucleation kinetics, as well as the overall kinetics of crystallization, can be considerably enhanced by flow [20-26]. Under certain conditions, even the crystal morphology will depend on the mechanical history. The result can be row-nucleated and shishkebab structures rather than the spherulitic structures typically encountered under quiescent conditions [25, 27-31]. Next to the thermomechanical history, the addition of fillers on the nucleation density can also be of importance in processing $[32,33]$, although this will not be the topic of this article. The effect of flow on the crystallization of thermoplastic elastomers (or segmented multiblock copolymers) has, to the best of our knowledge, not been investigated yet. In this article, the effect of thermomechanical history on the crystallization of commercial poly (ether-block-amide) is studied. The effect of temperature as well as the effect of shear flow will be considered.

\section{EXPERIMENTAL}

\section{Materials}

The poly(ether-block-amide) copolymer used here is a commercial Pebax ${ }^{\circledR}$ 7033SA (called PE- $b$-A hereafter) supplied by Arkema. It is a multiblock copolymer containing segments of polyamide 12 (PA12) and polytetramethylene glycol (PTMG). The sample as used here contains no additives but inherently contains catalyst traces. PA12 is a semicrystalline material with a glass transition temperature $T_{\mathrm{g}}$ of $36^{\circ} \mathrm{C}$ and an equilibrium melting temperature of $190^{\circ} \mathrm{C}$ [34]. PTMG on the other hand is amorphous at room temperature with its glass transition temperature around $-85^{\circ} \mathrm{C}$. The average molar mass $M_{\mathrm{n}}$ of the PE- $b$-A used in this study is about $20,000 \mathrm{~g} / \mathrm{mole}$. In order to compare the crystallization behavior of PE- $b$-A with its homopolymer equivalent, pure PA12 is selected with a comparable molar mass of $20,000 \mathrm{~g} / \mathrm{mole}$.

To investigate the morphology evolution in situ during crystallization, a wide variety of experimental techniques are available covering various length scales. Different sample preparation routes were followed according to the experimental technique used. Each procedure started with drying the polymer at $60^{\circ} \mathrm{C}$ before storing in a desiccator in the presence of silica gel.

\section{Experimental Methods}

In order to determine properly the transition temperatures in PE- $b$-A, screening experiments using DSC and $\mathrm{X}$-ray scattering have been performed. DSC measurements have been carried out on a TA Instruments Q2000 DSC under $\mathrm{N}_{2}$-atmosphere in order to avoid degradation. The SAXS apparatus used consisted of a $18 \mathrm{~kW}$ rotating-anode X-ray generator (Brucker Ax, Japan) with a graphite crystal monochromator to obtain a $\mathrm{Cu} \mathrm{K} \alpha$ beam with a wavelength of $0.154 \mathrm{~nm}$, and a one-dimensional position-sensitive proportional counter with line-focus optics. In order to record 2D WAXS patterns, an imaging plate was introduced. To complement these experiments, rheological measurements have been performed as well. For this purpose, a dynamic stress rheometer (Rheometrics Scientific) with a parallel plate geometry (25 mm diameter) and a gap thickness of $900 \mu \mathrm{m}$ has been employed.

The effect of thermomechanical history on crystallization is studied with a rheo-optical set up based on a Linkam CSS450 shearing system. Both microscopy and SALS experiments are performed. The SALS setup has a $10 \mathrm{~mW}$ polarized HeNe laser (wavelength $633 \mathrm{~nm}$ ) as a light source. Light from the laser is sent consecutively through a polarizer, and the sample and the analyzer is rotated either $90^{\circ}$ (HV mode) or $0^{\circ}$ (VV-mode) with respect to the first polarizer. Finally, the scattered light is recorded on a semitransparent screen, and the image is digitized and further processed by means of a home-built software (KULeuven-SALSSOFTWARE). For the optical microscopy experiments, the Linkam shearing cell is directly mounted on an optical microscope (Leitz Laborlux 12 Pol S). 


\section{RESULTS AND DISCUSSION}

\section{Determination of Transition Temperature}

PE- $b$-A is a multiblock copolymer which can-as indicated in the Introduction-exhibit quite a variety of microstructures. In order to process such a polymer properly or to perform crystallization experiments, the important transition temperatures such as the melting $\left(T_{\mathrm{m}}\right)$ and order-disorder temperatures $\left(T_{\mathrm{ODT}}\right)$ have to be determined. This can be achieved by means of various experimental methods. Here, a combination of rheology, DSC, and X-ray experiments (both WAXS and SAXS) have been used to determine both temperatures.

Using standard DSC-protocols, the melting temperatures were found to be $176^{\circ} \mathrm{C}$ for $\mathrm{PE}-b$-A and $182^{\circ} \mathrm{C}$ for PA12, respectively. The equilibrium melting temperature $T_{\mathrm{m} 0}$ of PE- $b$-A is determined with the Hoffman-Weeks protocol. In principle, $T_{\mathrm{m} 0}$ can be estimated by plotting $T_{\mathrm{m}}$ versus the inverse of the thickness of the crystalline layer and extrapolating to an infinite layer thickness. The crystalline layer thickness can for instance be determined by X-ray scattering experiments. According to Hoffman's theory of crystallization, the plot of $T_{\mathrm{m}}$ versus thickness should be linear. The procedure can be quite laborious since it requires extensive measurements of crystal thicknesses. The latter can be overcome by means of the Hoffman-Weeks approach, based on the difference between the melting temperature and the crystallization temperature [35]. This method is known to yield values of $T_{\mathrm{m} 0}$ similar to those derived from varying the crystal thickness [36]. Although originally developed for homopolymers, the Hoffman-Weeks method has successfully been used for multiblock copolymers as well [37-39]. The resulting value for the equilibrium melting temperature of PE- $b$-A is calculated to be $184^{\circ} \mathrm{C}$. This value is lower than the reported equilibrium melting temperature of $190^{\circ} \mathrm{C}$ for PA12 [34]. Block copolymers however can be expected to melt at a lower temperature than the crystalline homopolymer as was discussed by Flory and Mandelkern [40], an effect associated to the presence of steric hindrance of the second component, which avoids the formation of more perfect crystalline structures.

Since PE- $b$-A has been reported to exhibit a microphase-separated structure [15], it is important to know the so-called order-disorder temperature $T_{\mathrm{ODT}}$, which marks the limit between organized and unorganized states in the phase diagram. Its value is required in order to establish the experimental protocol for the crystallization experiments (see further). Assuming for instance that $T_{\text {ODT }}$ is above the melting temperature $T_{\mathrm{m}}$ of the hard blocks, even the molten material would still display a microstructure, albeit on a very local scale. Various methods have been proposed to determine $T_{\text {ODT }}$ [41-46], of which rheometry and SAXS measurements have been adopted in this work. The first one was proposed by Han et al. [42] and has proven to be adequate in determining the $T_{\text {ODT }}$ of

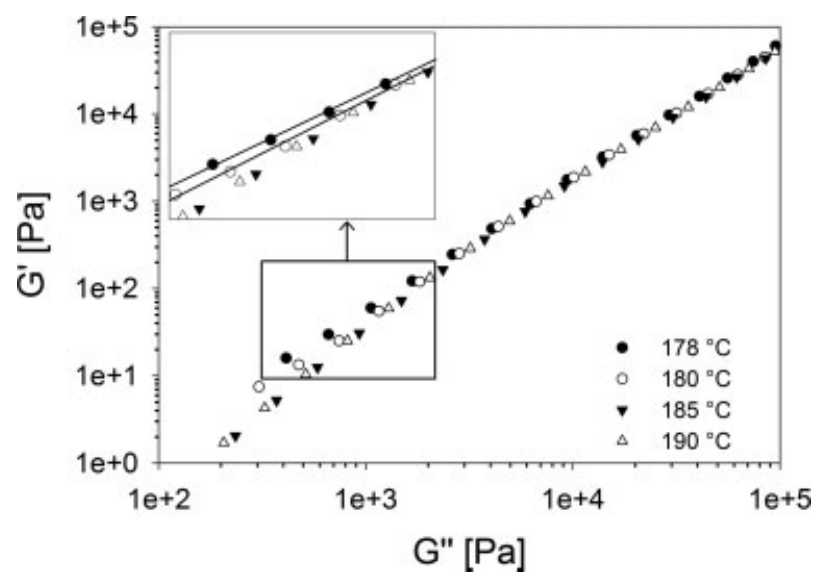

FIG. 1. $G^{\prime}$ versus $G^{\prime \prime}$ curves at different temperatures. The inset gives a detailed view of the deviations from the master curve.

other multiblock copolymers such as poly(ether-ester) multiblock copolymers [47]. The method is based on linear viscoelastic measurements: a change in the $G^{\prime}$ versus $G^{\prime \prime}$ curves is expected at the temperatures at which the microstructure changes. This temperature can then be regarded as the $T_{\text {ODT }}$. The result of applying this method to determine $T_{\text {ODT }}$ in PE- $b-\mathrm{A}$ is shown in Fig. 1 . In this figure, it can be seen that plots of $G^{\prime}$ versus $G^{\prime \prime}$ coincide as long as the temperature is above $185^{\circ} \mathrm{C}$, indicating that above this temperature, no specific microstructure is present. At $180^{\circ} \mathrm{C}$, however, the curve does not superimpose anymore on those recorded at higher temperatures, a feature that is even more pronounced at $178^{\circ} \mathrm{C}$. These temperatures are still above the melting temperature of the PA-blocks, indicating that the $T_{\text {ODT }}$ of PE- $b$-A is in the temperature interval of $180-185^{\circ} \mathrm{C}$.

To verify the measured value of $T_{\mathrm{ODT}}$, additional SAXS experiments have been performed. The material was first annealed at $200^{\circ} \mathrm{C}$ to erase the earlier thermomechanical history. Subsequently, the material was quenched to $150^{\circ} \mathrm{C}$ and was allowed to crystallize. After this solidification, the X-ray signals were recorded. Once the data acquisition was complete, the temperature was increased to $178^{\circ} \mathrm{C}$ (heating rate $5^{\circ} \mathrm{C} / \mathrm{min}$ ). After waiting for 30 min, the scattering intensities were recorded for another $30 \mathrm{~min}$ in order to obtain the SAXS pattern at this particular temperature. A similar protocol was applied at all subsequent temperatures, although smaller temperature increments were used to achieve sufficient resolution in determining $T_{\mathrm{ODT}}$.

The SAXS profiles resulting from this protocol are shown in Fig. 2. At $150^{\circ} \mathrm{C}$, a clear peak at $q=0.32$ $\mathrm{nm}^{-1}$ is observed, associated with the lamellar spacing inside the crystals. This peak has disappeared at $178^{\circ} \mathrm{C}$ (i.e., above the melting temperature of the PA12-blocks), but a significant SAXS profile remains above $178^{\circ} \mathrm{C}$. At temperatures above $185^{\circ} \mathrm{C}$, the SAXS signal disappears, indicating that no microstructure is present anymore. Based on these measurements, it can be concluded that 


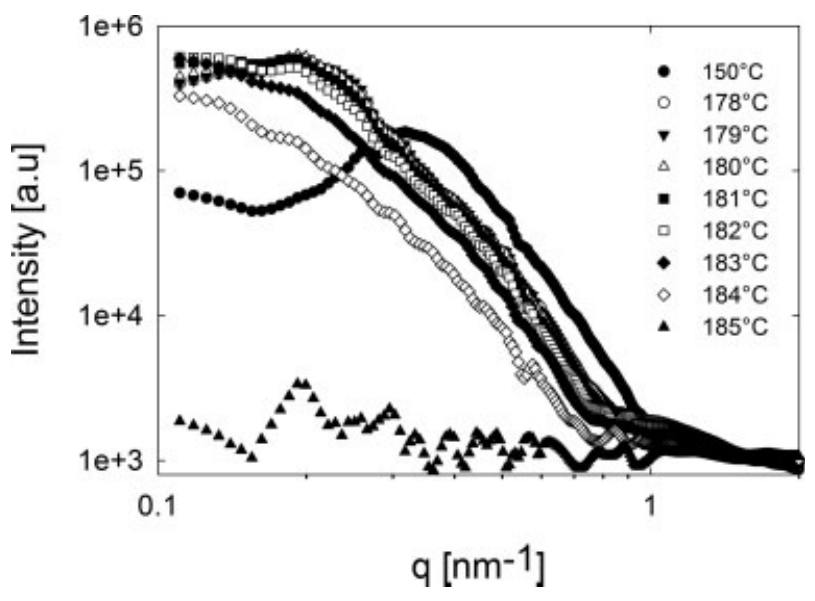

FIG. 2. SAXS profiles for different temperatures during a heating scan, starting at $150^{\circ} \mathrm{C}$.

the order-disorder temperature is at $185^{\circ} \mathrm{C}$, consistent with the rheological measurements.

\section{Quiescent Crystallization}

In this section, the quiescent crystallization behavior of PE- $b$-A is studied by means of optical microscopy and SALS. The SALS patterns have been analyzed according to the method developed by Stein and coworkers [48-50]. In this method, the evolution of the scattering patterns is described in terms of the density invariant $Q_{\eta}$ and the orientation invariant $Q_{\delta}$ that are defined as integrated scattering intensities:

$$
\begin{gathered}
Q_{\eta}=\int_{0}^{\infty}\left(I_{\mathrm{VV}}-\frac{4}{3} I_{\mathrm{HV}}\right) q^{2} . \\
Q_{\delta}=\int_{0}^{\infty} I_{\mathrm{HV}} q^{2} d q .
\end{gathered}
$$

As can be seen in Eqs. 1 and 2, both $\mathrm{HV}$ as well as VV experiments need to be performed in order to calculate the invariants. Both invariants can also be expressed in function of the polarizabilities of the crystalline and the amorphous phases [49]. Basically, the density invariant $Q_{\eta}$ is proportional to the difference in polarizability between the growing crystalline phase and the surrounding amorphous melt. The orientation invariant $Q_{\delta}$ is determined by the contrast in polarizabilities inside the growing crystalline phase. This method has been used extensively to describe the crystallization of polymersboth in the quiescent as well as in the flow-induced state- and will be adopted here as well. A general overview of this approach in the study of polymer crystallization can be found in a recent review by Baert and Van Puyvelde [51].

The experimental protocol consists in first annealing the sample at $200^{\circ} \mathrm{C}$ for $5 \mathrm{~min}$ in order to erase the earlier thermomechanical history. This temperature is above the order-disorder temperature since the crystallization experiments are performed starting from an amorphous melt. Subsequently, the material is cooled down at a rate of $30^{\circ} \mathrm{C} / \mathrm{min}$ to the desired isothermal crystallization temperature. As was stated in Experimental section, these experiments are performed both on PE- $b-\mathrm{A}$ and pure PA12.

The optical micrographs (not shown) displayed a spherulitic morphology, both for the homopolymer as well as for the multiblock. However, the spherulites of the PE$b$-A were less well defined, since the flexible PTMG segments introduce defects in the crystalline structure. This was reflected in the absence of a Maltese cross in a crossed polarizer microscopy experiment. With the optical microscopy, the spherulitic growth rates have been determined. These are shown as a function of temperature in Fig. 3. Since the experiments are conducted close to the melting temperature, i.e., at a low degree of undercooling, an increase in growth rate with increasing degree of undercooling is expected. The growth rate of the pure PA12 with similar molar mass turned out to be higher than the growth rates measured in PE- $b$-A, but they both depend in a similar fashion on temperature. When comparing the data at the same degree of undercooling (see Fig. 4), the growth rates of the pure PA12 and PE- $b$-A (i.e., the PA12 blocks in the PE- $b$-A) superimpose. Hence, under these conditions, spherulites in the multiblockwhich consists of $88 \%$ in weight of PA12-grow at a similar rate as their homopolymer equivalent.

Typical SALS-patterns, both in HV and in VV-modeduring the crystallization of PE- $b$-A are presented in Fig. 5. As expected, the VV measurements show a circular symmetry, indicating that the isotropic structures do not have any preferential orientation. Typical for the presence of spherulites, the HV experiments show a four-lobe pattern. The lobes are not very well pronounced, consistent with the extremely low contrast within the spherulites in crossed polarizer microscopy experiments.

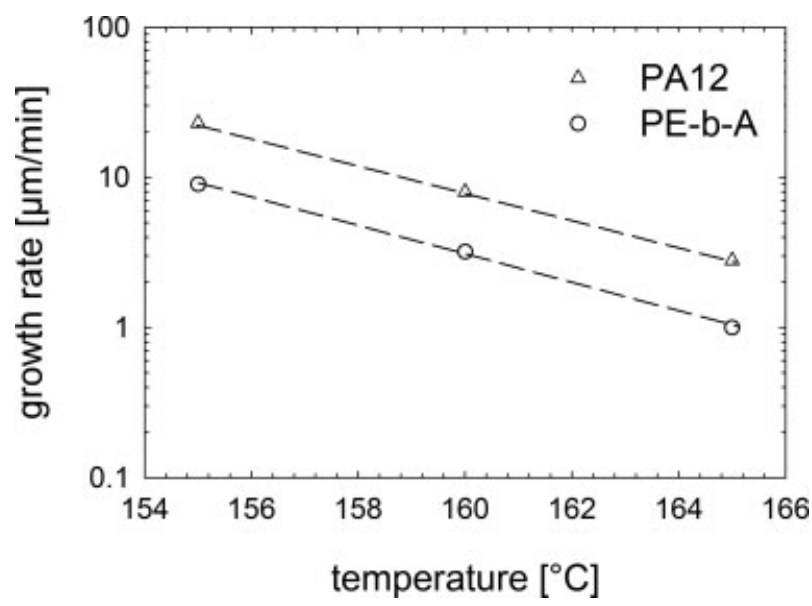

FIG. 3. Spherulitic growth rates as a function of temperature for PA12 and PE- $b$-A. The dashed line is to guide the eye. 


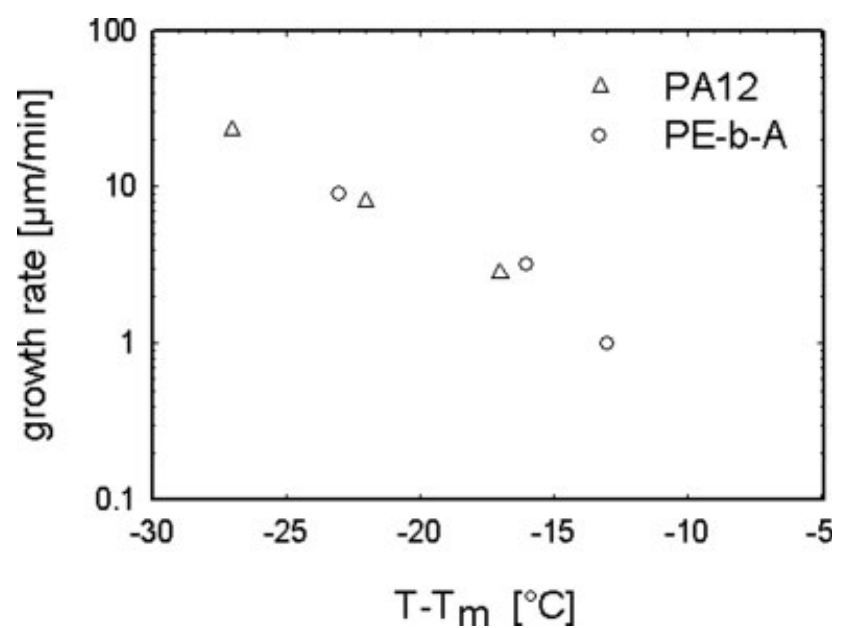

FIG. 4. Spherulitic growth rates for PA12 and PE- $b$-A as a function of degree of undercooling.

In order to quantify the crystallization kinetics, the scattering invariants are calculated according to Eqs. 1 and 2. The results for the PA12 are shown in Fig. 6. Both invariants show the typical evolution observed for many polymers [51]. Here, the two invariants start to increase almost simultaneously. This is not universal since in many cases a delay between the onset of growth of the density and orientation invariants has been reported (see [51] for an overview). As was already discussed earlier, the density invariant is determined by the scattering contrast between the growing crystalline phase and the surrounding amorphous melt. On the other hand, the orientation invariant is determined by the internal crystalline scattering contrast, a simultaneous increase is not guaranteed and requires specific material characteristics.

The time at which the scattering functions appear depends on the crystallization temperature (Fig. 6), reflecting the temperature dependence of the crystallization kinetics.

Figure 7 shows the scattering invariants for PE- $b-\mathrm{A}$. The evolution of the scattering invariants for the multiblock is similar to that found in the homopolymer. In both cases, the onset times of the scattering and the density

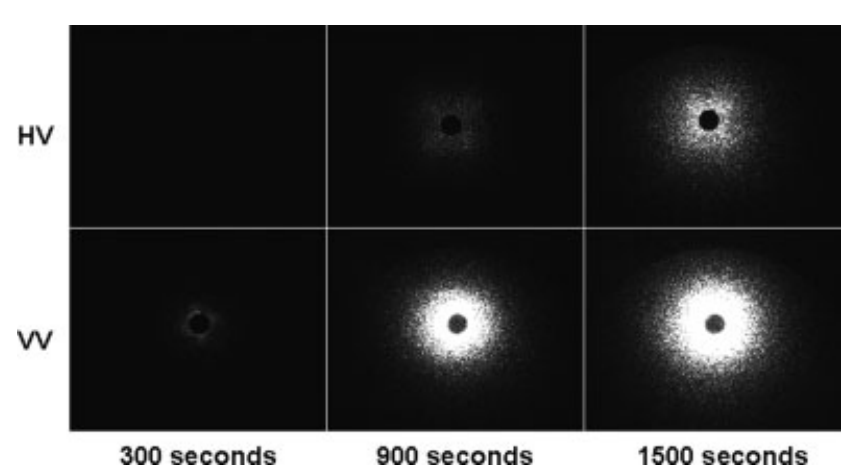

FIG. 5. Evolution of the VV and HV scattering patterns of PE- $b$-A during an isothermal crystallization at $165^{\circ} \mathrm{C}$.

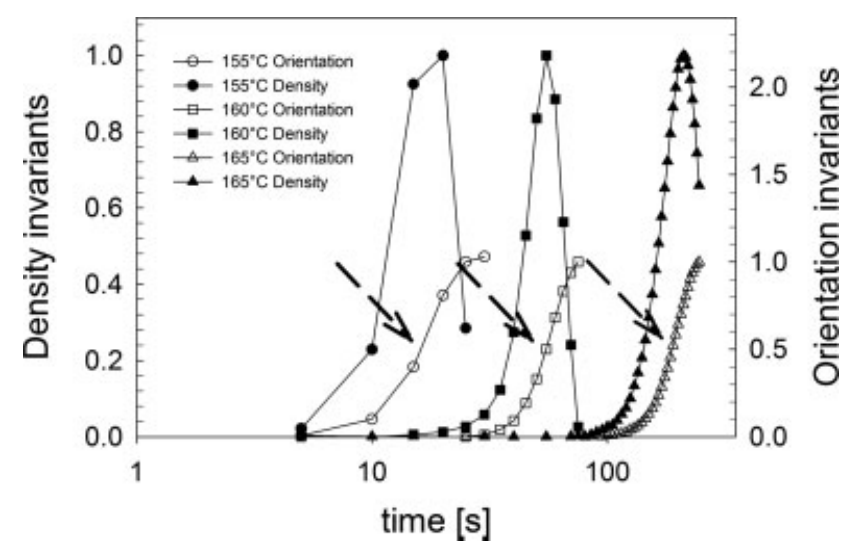

FIG. 6. Effect of temperature on the density and orientation invariant during isothermal crystallization of PA12.

invariants slightly differ especially at a larger degree of undercooling. In order to compare the crystallization kinetics, the inflection point of the orientation invariant is taken as a characteristic crystallization time. The crystallization kinetics of PA12 and PE- $b$-A, at the same degree of undercooling, is compared in Fig. 8. Taking into account the relatively large uncertainty on the time scale for PE- $b$-A due to residual catalyst traces that can influence the nucleation rate, it can be concluded that the hard copolymer studied in this work (88\% PA12) has a crystallization time that is quite similar with that of its homopolymer equivalent.

\section{Flow-Induced Crystallization}

During processing, polymers are sometimes subjected to quite extreme flow conditions. Shear can have a profound effect on the crystallization process, ranging from an increase in nucleation rate to even modification of the crystalline morphology (see Introduction section). At present, however, no studies on the effect of flow on the crystallization of segmented multiblocks such as PE- $b$-A seem to have been conducted. Here, the experimental protocol

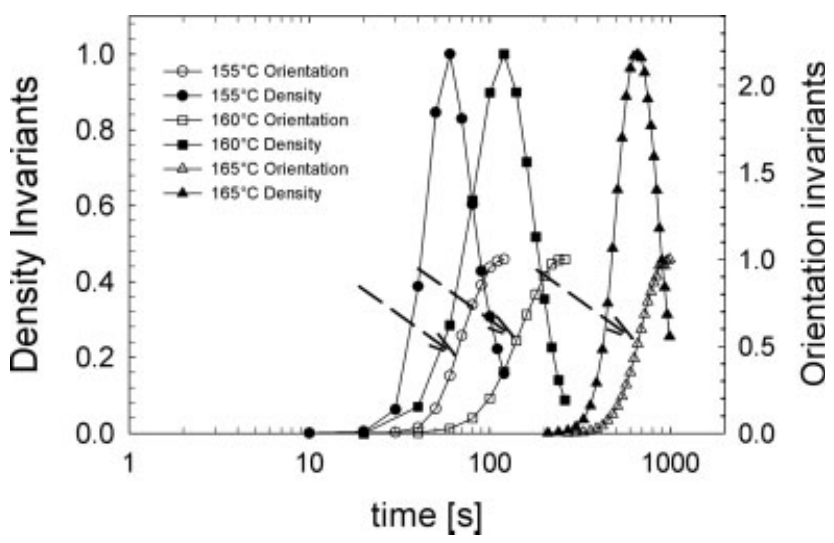

FIG. 7. Effect of temperature on the density and orientation invariant during isothermal crystallization of PE- $b$-A. 


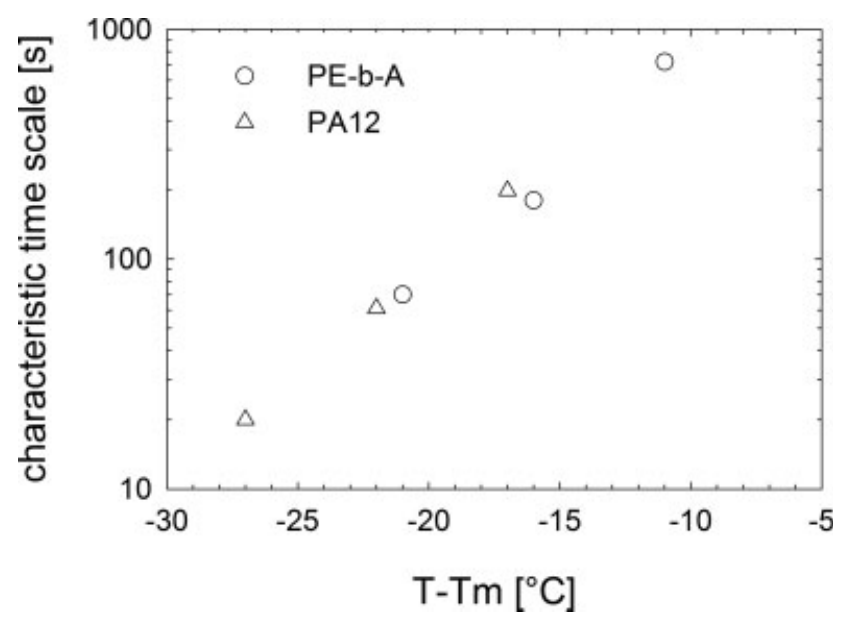

FIG. 8. Comparison of the characteristic crystallization time scales as a function of the degree of undercooling for PA12 and PE- $b-\mathrm{A}$.

developed by Janeschitz-Kriegl has been adopted [24]. The material is first heated to $200^{\circ} \mathrm{C}$ for $5 \mathrm{~min}$ to erase the thermomechanical history, after which the sample is cooled at $30^{\circ} \mathrm{C} / \mathrm{min}$ to the desired crystallization temperature. Once the crystallization temperature is reached, a short shear pulse is applied. The shear rate and the shearing time can be varied independently. A constant total strain $\gamma$ (i.e., shear rate $\times$ shearing time) of 50 is chosen. The effect of shear flow on the crystallization process is investigated again with optical microscopy and SALS.

In Fig. 9, the nucleation densities in PA12 and PE- $b$ A, as derived from optical microscopy experiments at the same degree of undercooling $\left(20^{\circ} \mathrm{C}\right)$, are compared as a function of shear rate (at constant $\gamma=50$ ). The number of nuclei in PA12 is drastically enhanced when the shear rate is increased. This feature is commonly observed in many homopolymer systems and is associated with the flow-induced orientation of the chain. The probability of achieving the energetically stable unit cell conformation is clearly smaller in the random coil state in quiescent

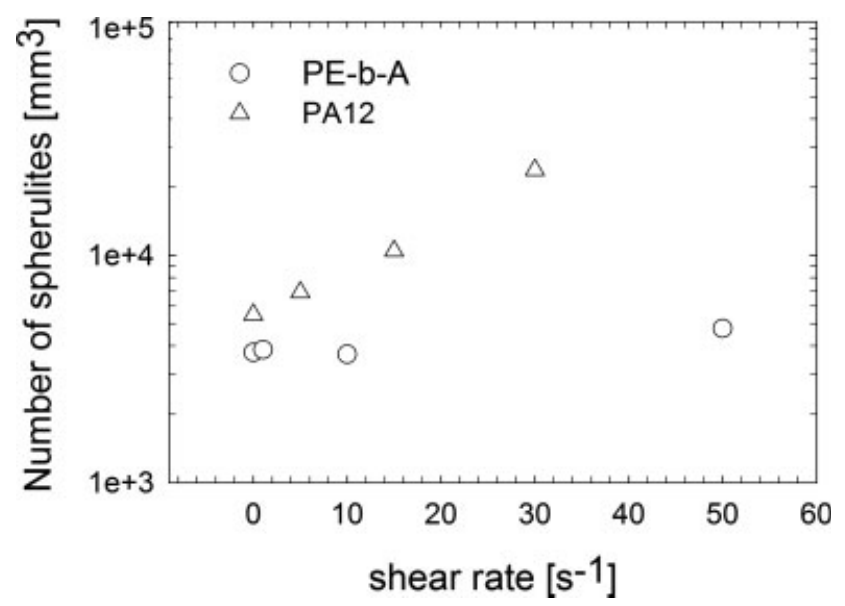

FIG. 9. Nucleation density as a function of shear rate for PA12 and PE- $b$-A ( $\gamma=50$, same degree of undercooling). conditions than when oriented by flow. For the PE- $b-\mathrm{A}$ material, the effect of shear rate on the nucleation density is negligible, at least in the conditions studied here. Despite the fact that the imposed shear flow orients the segmented multiblock, no significant additional nuclei are created. Based on rheological data (linear viscoelastic measurements), the longest relaxation time at this particular temperature is estimated to be $1 \mathrm{sec}$ for both PA12 and PE- $b$-A. Hence, the Deborah number De-a measure typically used to determine the onset of molecular orientation (see for instance [22])_exceeds a value of unity at shear rates above $1 \mathrm{sec}^{-1}$. On this basis, an enhanced nucleation density would be expected in both polymers. As can be seen in Fig. 9, this is not the case for PE- $b$-A. The precise reason for this phenomenon is not fully clear at present, but it is most probably related to the presence of catalyst traces that serve as heterogeneous nuclei.

The total kinetics of the crystallization process after different shear histories is shown in Fig. 10. Only the orientation invariant is shown here, since the inflection point on this curve is used as the characteristic crystallization time. As can be seen, flow does not have an effect on the crystallization time scale, since within experimental error, all curves superimpose. A comparison between the characteristic time scales_-defined as the inflection point of the orientation invariant as a function of shear rate for PE- $b-\mathrm{A}$ and PA12-is shown in Fig. 11. In this figure, it can be seen that the effect on the kinetics is more pronounced on the homopolymer since the characteristic time scale decreases substantially with shear rate.

The dependence of the characteristic crystallization time for PA12 as a function of shear rate is roughly comparable with the dependence of its nucleation density on shear rate. This indicates that the subsequent growth rates of PA12 crystals are not significantly influenced by the preceding flow, as could be expected. Hence, from the data, it can be concluded that the increase in crystallization speed in PA12 is essentially dominated by the increase in nucleation density. In the case of PE- $b-\mathrm{A}$, the characteristic crystallization rate is independent of the

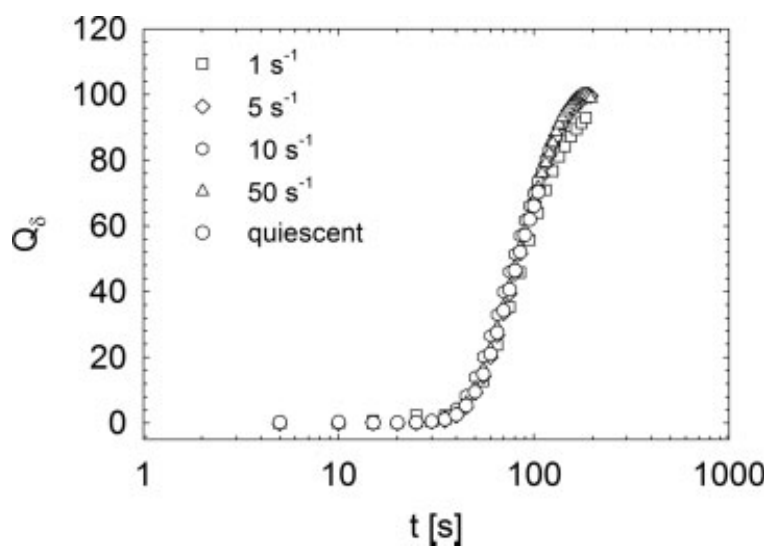

FIG. 10. Effect of shear rate (total strain $=50$ ) on the scattering invariants during an isothermal crystallization of PE- $b$-A at $156^{\circ} \mathrm{C}$. 


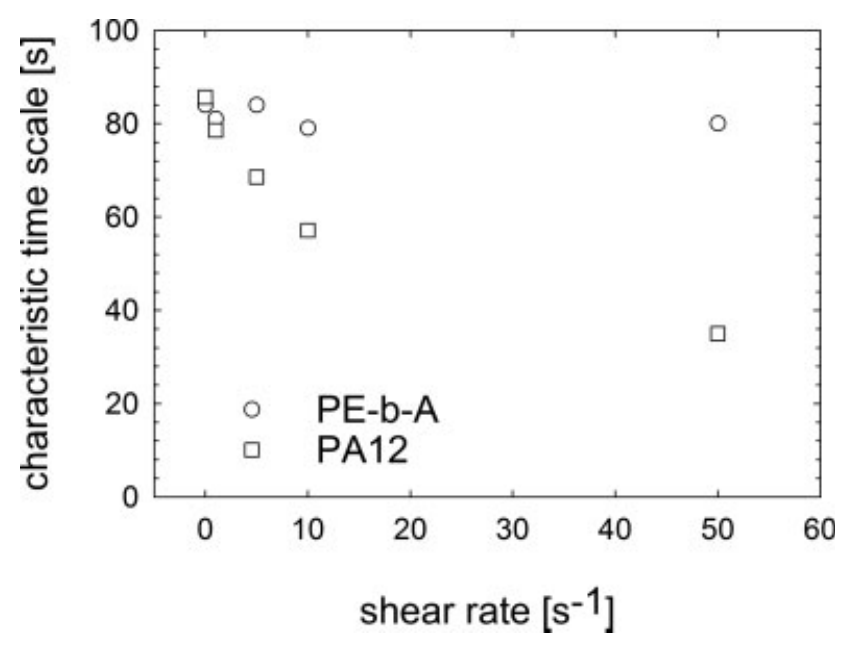

FIG. 11. Characteristic crystallization times as a function of shear rate for PA12 and PE- $b$-A at an equal degree of undercooling $\left(20^{\circ} \mathrm{C}\right)$.

shear conditions, at least in the experimental window studied here. This is consistent with the absence of a shear effect on the nucleation density.

\section{CONCLUSIONS}

In this work, both the quiescent and shear-induced crystallization of a poly(ether-block-amide) and pure PA12 of equal molar mass are studied by means of optical microscopy and SALS. In order to define a proper experimental protocol, the melting and order-disorder temperatures have been determined. The latter was studied both by rheology and X-ray measurements. In the quiescent case, the poly(ether-block-amide), containing 88 wt $\%$ of PA12 and very low molar mass PTMG segments, shows a crystallization behavior very similar to that of its homopolymer equivalent, both regarding the spherulitic growth rates as well as the total kinetics. The spherulitic morphology, however, is less developed in the block copolymer because of the presence of the PTMG segments that introduce defects in the crystalline ordering. After application of flow, the situation becomes different. In the case of PA12, flow increases both the nucleation density and the global kinetics of the process. In the case of poly(ether-block-amide) studied in this article, flow did not have a significant effect on the nucleation density possibly because of the presence of a large amount of heterogeneous nucleation sites. The total kinetics of the crystallization process was not enhanced by flow, at least under the conditions studied here.

\section{REFERENCES}

1. N.R. Legge, G. Holden, and H.E. Schroeder, Thermoplastic Elastomers: A Comprehensive Review, Hanser, Munich (1987).

2. D. Nichetti and N. Grizzuti, Polym. Eng. Sci., 44, 1514 (2004).
3. D.W. Lee, S.H. Lee, S.C. Kim, K.H. Char, J.H. Park, and Y.H. Bae, J. Polym. Sci. B. Polym. Phys., 41, 2365 (2003).

4. P.R. Laity, J.E. Taylor, S.S. Wong, P. Khunkamchoo, K. Norris, M. Cable, G.T. Andrews, A.F. Johnson, and R.E. Cameron, Polymer, 45, 7273 (2004).

5. W. Gabrielse, M. Soliman, and K. Dijkstra, Macromolecules, 34, 1685 (2001).

6. F.S. Bates, and G.H. Fredrickson, Annu. Rev. Phys. Chem., 41, 525 (1990).

7. V. Castelletto and I.W. Hamley, Curr. Opin. Solid State Mater. Sci., 8, 426 (2004).

8. I.W. Hamley and V. Castelletto, Prog. Polym. Sci., 29, 909 (2004).

9. E. Helfand and J.I. Lauritze, Macromolecules, 6, 631 (1973).

10. V.I. Bondar, B.D. Freeman and I. Pinnau, J. Polym. Sci. B. Polym. Phys., 37, 2463 (1999).

11. M.L. Di Lorenzo, M. Pyda, and B. Wunderlich, J. Polym. Sci. B. Polym. Phys., 39, 1594 (2001).

12. M. Xie and Y. Camberlin, Makromol. Chem., 187, 383 (1986).

13. B.B. Sauer, R.S. McLean, D.J. Brill, and D.J. Londono, J. Polym. Sci. B. Polym. Phys., 40, 1727 (2002).

14. B.B. Sauer, R.S. McLean, and R.R. Thomas, Polym. Int., 49, 449 (2000).

15. J.P. Sheth, J.N. Xu, and G.L. Wilkes, Polymer, 44, 743 (2003).

16. E. Okoroafor and J. Rault, J. Polym. Sci. B. Polym. Phys., 29, 1427 (1991).

17. S. Ghosh, D. Khastgir, and A.K. Bhowmick, Polymer, 39, 3967 (1998).

18. I.K. Yang, and P.H. Tsai, J. Central South Univ. Technol., 14, 146 (2007).

19. I.K. Yang and P.H. Tsai, J. Polym. Sci. B. Polym. Phys., 43, 2557 (2005).

20. G. Kumaraswamy, J. Macromol. Sci. Polym. Rev., C45, 375 (2005).

21. J.A. Kornfield, G. Kumaraswamy, and A.M. Issaian, Ind Eng. Chem. Res., 41, 6383 (2002).

22. S. Acierno, S. Coppola, N. Grizzuti, and P.L. Maffettone, Macromol. Symp., 185, 233 (2002).

23. N. Devaux, B. Monasse, J.M. Haudin, P. Moldenaers, and J. Vermant, Rheol. Acta., 43, 210 (2004).

24. H. Janeschitz-Kriegl, Colloid Polym. Sci., 281, 1157 (2003).

25. J. Baert, P. Van Puyvelde, and F. Langouche, Macromolecules, 39, 9215 (2006).

26. J. Baert and P. Van Puyvelde, Polymer, 47, 5871 (2006).

27. H.E.H. Meijer, Materials Science and Technology: A Comprehensive Treatment, Vol. 18, Wiley, Weinheim (1997).

28. J.K. Keum, F. Zuo, and B.S. Hsiao, J. Appl. Crystallogr., 40, S48 (2007).

29. S. Kimata, T. Sakurai, Y. Nozue, T. Kasahara, N. Yamaguchi, T. Karino, M. Shibayama, and J.A. Kornfield, Science, 316, 1014 (2007).

30. M. Muthukumar, "Modeling Polymer Crystallization," in Interphases and Mesophases in Polymer Crystallization III, Vol. 191, Springer, Berlin, 241 (2005). 
31. R.H. Somani, L. Yang, L. Zhu, and B.S. Hsiao, Polymer, 46, 8587 (2005).

32. C. Deshmanea, Q. Yuan, R.S. Perkins, and R.D.K. Misra, Mater. Sci. Eng. A., 458, 150 (2007).

33. Q. Yuan, S. Awate, and R.D.K. Misra, J. Appl. Polym. Sci., 102, 3809 (2006).

34. C.J.G. Plummer, J.E. Zanetto, P.E. Bourban, and J.A.E. Manson, Colloid Polym. Sci., 279, 312 (2001).

35. J.D. Hoffman and J.J. Weeks, J. Chem. Phys., 32, 1723 (1962).

36. E.B. Bond and J.E. Spruiell, J. Appl. Polym. Sci., 81, 229 (2001).

37. C.L. Lewis, and J.E. Spruiell, J. Appl. Polym. Sci., 100, 2592 (2006).

38. J. Cao, W. Su, Z. Wu, J. Liu, T. Kitayama, and K. Hatada, Polymer, 37, 4579 (1996).

39. M. Vannini, L. Finelli, N. Lotti, M. Colonna, C. Lorenzetti, and A. Munari, J. Polym. Sci. B Polym. Phys., 43, 1441 (2005).

40. P.J. Flory and L. Mandelkern, J. Polym. Sci., 21, 345 (1956).
41. C. Burger, T. Wolff, and W. Ruland, Abstr. Papers Am. Chem. Soc., 207, 155 (1994).

42. C.D. Han, D.M. Baek, J.K. Kim, and S.G. Chu, Polymer, 33, 294 (1992).

43. T. Hashimoto, T. Ogawa, and C.D. Han, J. Phys. Soc. Jpn., 63, 2206 (1994).

44. J.K. Kim, H.H. Lee, Q.J. Gu, T.H. Chang, and Y.H. Jeong, Macromolecules, 31, 4045 (1998).

45. J.H. Rosedale and F.S. Bates, Macromolecules, 23, 2329 (1990).

46. B. Stuhn, R. Mutter, and T. Albrecht, Europhys. Lett., 18, 427 (1992).

47. H. Veenstra, R.M. Hoogvliet, B. Norder, and A.P. de Boer, J. Polym. Sci. B Polym. Phys., 36, 1795 (1998).

48. J. Koberstein, T.P. Russell, and R.S. Stein, J. Polym. Sci. B Polym. Phys., 17, 1719 (1979).

49. R.S. Stein and P.R. Wilson, J. Appl. Phys., 33, 1914 (1962).

50. D.Y. Yoon, and R.S. Stein, J. Polym. Sci. B Polym. Phys., 12, 735 (1974).

51. J. Baert and P. Van Puyvelde, Macromol. Mater. Eng., 293(4), 255 (2008). 